\title{
Structure-based characterization and optimization of Eph receptor-targeting peptides
}

\author{
Bernhard C. Lechtenberg ${ }^{1 \#}$, Philip E. Dawson ${ }^{2}$ Elena B. Pasquale ${ }^{1}$, and Stefan J. Riedl ${ }^{1}$
}

${ }^{1}$ Sanford Burnham Prebys Medical Discovery Institute, La Jolla, CA, USA

2 The Scripps Research Institute, La Jolla, CA, USA

\#Contact: blechtenberg@sbpdiscovery.org

Eph receptors are a 14-member family of membrane-spanning receptor tyrosine kinases that mediate bidirectional communication between cells, with many roles in human physiology, including cell adhesion, proliferation, survival, differentiation, and self-renewal. Accordingly, Eph receptors play roles in a diverse set of diseases, including neurodegenerative diseases (e.g. ALS and Alzheimer's), recovery after stroke, and cancers. Eph receptors are activated by promiscuous interaction with one of the 8 cell surface-associated Ephrin ligands at cell-cell contacts. Ephrins bind to the extracellular Eph ligand-binding domain (LBD), leading to receptor clustering, autophosphorylation of the cytosolic kinase domain, and interaction with downstream signaling effectors. Eph receptors have emerged as promising drug targets, for example by blocking binding of the Ephrins to the extracellular LBD. However, the large and flexible extracellular binding pocket has proven difficult to target with small molecules.

Thus, our approach was to use a phage-display peptide library to identify peptides that potently and specifically bind to the extracellular EphA4 LBD as potential drug leads for the treatment of ALS, Alzheimer's disease, and breast cancer. The circular (disulfide-containing) peptide APYCVYRGSWSC (APY) specifically binds to EphA4, but not to other Eph receptors, with a modest in vitro IC 50 of $2.7 \mu \mathrm{M}$. APY is an EphA4 antagonist and inhibits binding of EphrinA5 and downstream signaling. We successfully solved the structure of the EphA4 LBD/APY complex and used structure-guided chemical biology to improve the potency and plasma-stability of the APY peptide. Our current lead compound APY-d3 ([ $\beta$-Ala]PYCVYR[ $\beta$-Ala]SWSC-NH ${ }_{2}$ ) shows a more than 100-fold improved potency (IC 50 of $19 \mathrm{nM}$ in vitro, IC 50 of $240 \mathrm{nM}$ in cells) and outstanding plasma stability ( $t_{1 / 2}>72$ hours) while maintaining specificity for EphA4. Our current efforts focus on improving kidney clearance in vivo and to establish animal efficacy studies.

In a second study, we identified the peptide YSAYPDSVPMMS (YSA) that specifically targets EphA2 with an affinity of about $3 \mu \mathrm{M}$. Different to the antagonistic EphA4-specific peptide APY, YSA is an agonistic peptide and induces EphA2 autophosphorylation. YSA derivatives have been used for directed delivery of chemotherapeutics in various cancer models in the literature. Previous efforts to improve stability and potency of YSA have proven difficult, mainly due to lack of structural information. We recently solved the crystal structure of the EphA2 LBD/YSA complex and will use this structure to guide a chemical biology approach to improve potency and plasma stability of the YSA peptide.

Our studies show how peptides and peptide-derivatives can be used to specifically and potently target Eph receptors and highlight the strengths of our structure-guided chemical biology optimization strategy. 